INPLASY

PROTOCOL

To cite: Tang et al. Herbal medicine for relapse and metastasis in patients operated for colorectal cancer: a systematic review and meta-analysis. Inplasy protocol 2020120127. doi: 10.37766/inplasy2020.12.0127

Received: 25 December 2020

Published: 25 December 2020

Corresponding author:

Mo Tang

904608946@qq.com

Author Affiliation:

China Academy of Chinese

Medical Science

Support: NATCM.

Review Stage at time of this submission: The review has not yet started.

Conflicts of interest: None.

\section{Herbal medicine for relapse and metastasis in patients operated for colorectal cancer: a systematic review and meta-analysis}

\author{
Tang, M1; He, B2; Yang, Y3; Wu, N4.
}

Review question / Objective: Participants: Patients diagnosed with Colorectal cancer (pathologically diagnosis of any tumor stage except for stage D/stage IV) and undergoing radical surgery, regardless gender, age or race. Interventions: Herbal medicine (single herb or compound recipe), regardless administration route (orally, intravenously, or local administration such as clysis), Comparison: - herbal medicine versus no-intervention - herbal medicine versus chemotherapy or radiotherapy - herbal medicine combined with radio-chemotherapy versus chemotherapy or radiotherapy alone Outcomes The primary outcomes: disease-free survival and recurrence and metastasis rate. Secondary outcomes: Overall survival, quality of life and adverse reactions rate. Studies Randomized or quasirandomized trial will be considered regardless of blinding. Non-randomized controlled studies will be included in exploratory analysis.

INPLASY registration number: This protocol was registered with the International Platform of Registered Systematic Review and Meta-Analysis Protocols (INPLASY) on 25 December 2020 and was last updated on 25 December 2020 (registration number INPLASY2020120127).

\section{INTRODUCTION}

Review question / Objective: Participants: Patients diagnosed with Colorectal cancer (pathologically diagnosis of any tumor stage except for stage D/stage IV) and undergoing radical surgery, regardless gender, age or race. Interventions: Herbal medicine (single herb or compound recipe), regardless administration route (orally, intravenously, or local administration such as clysis), Comparison: - herbal medicine 
versus no-intervention - herbal medicine versus chemotherapy or radiotherapy herbal medicine combined with radiochemotherapy versus chemotherapy or radiotherapy alone Outcomes The primary outcomes: disease-free survival and recurrence and metastasis rate. Secondary outcomes: Overall survival, quality of life and adverse reactions rate. Studies Randomized or quasi-randomized trial will be considered regardless of blinding. Nonrandomized controlled studies will be included in exploratory analysis.

Condition being studied: Colorectal cancer (CRC) ranks third in terms of incidence and second in terms of cancer-related mortality worldwide. Due to the considerable changes in lifestyle and diet, CRC has become one of the rapidly increasing malignant tumors in China. It projected that the incidence will reach 20.7 per 100,000 in 2020. Once the CRC is detected, curative surgery is the treatment of choice for nonmetastatic CRC, Postoperative recurrence and metastasis are the most important factors that affect CRC patients' survival. Traditional Chinese Medicine(TCM) is deeply rooted in Chinese culture, where it has been used for thousands of years and is covered by most Chinese health insurance, many Chinese patients use TCM during or after aggressive cancer treatment.Studies have reported that TCM treatment has a protective effect on the long-term prognosis of postoperative patients with $\mathrm{CRC}$, resulting in improved survival.

\section{METHODS}

Participant or population: Patients diagnosed with Colorectal cancer (pathologically diagnosis of any tumor stage except for stage D/stage IV) and undergoing radical surgery, regardless gender, age or race.

Intervention: Herbal medicine (single herb or compound recipe), regardless administration route (orally, intravenously, or local administration such as clysis).
Comparator: - Herbal medicine versus nointervention; - herbal medicine versus chemotherapy or radiotherapy; - herbal medicine combined with radiochemotherapy versus chemotherapy or radiotherapy alone.

Study designs to be included: Randomized or quasi-randomized trial will be considered regardless of blinding. Nonrandomized controlled studies will be included in exploratory analysis.

Eligibility criteria: Meet the requirements of PICOS.

Information sources: 1.Electronic searching: Using Cochrane Colorectal Cancer Group search strategy to retrieve primary trials from the Cochrane Library, MEDLINE, EMBASE, CBM (Chinese Biological Medicine Database), CMCC (Chinese Medical Current Contents) and BIOSIS database since their start publication. The search key words for searching for databases above are as follows: 1) colorectal cancer, colonic cancer, rectal cancer. 2) traditional Chinese medicine, Chinese herbal medicine plant extract, herbal medicine Furthermore, unpublished trial reports from academic conferences, and theses of postgraduates are included in the range of manual searching.

Main outcome(s): The primary outcomes were disease-free survival and incidence of relapse and/or metastasis. With overall survival,quality of life and incidence of adverse reactions secondary outcomes.

Quality assessment / Risk of bias analysis: According to the Cochrane risk of bias tool (Cochrane Handbook for Systematic Reviews of Interventions, version 5.1.0),21,22 two investigators independently examined the quality of all included trials. Discrepancies were resolved either by consensus or through adjudication by a third investigator. The quality evaluation items of each trial included selection bias (random sequence generation and allocation concealment), performance bias (blinding of participants and personnel), 
detection bias (blinding of outcome assessment), attrition bias (incomplete outcome data), reporting bias (selective reporting), and other bias. These items were scored as low, high, or unclear risk of bias.

Strategy of data synthesis: No matter the administration modes, dosage and treatment duration, each herbal medicine would be independently compared to a control. Same herbal medicine compared with the same control, meta-analysis would be used to present their comparison. The dichotomous data will be presented as relatively risk (RR), continuous variance adopts weighted mean difference, both expressed by the 95 percent confidence interval $(\mathrm{Cl})$. Analyses are performed by intention-to-treat where possible. For dichotomous outcomes, patients with incomplete or missing data will be included in a sensitivity analysis by counting them as treatment failures to explore the possible effect of loss to follow-up on the findings ('worst-case' scenario). Heterogeneity will be tested for using Chi square with significance being set at $p<$ 0.10 . Whenever there is significant heterogeneity, the random effects model will be used. Meta-analysis will be performed using the Cochrane software, RevMan Analysis, in Review Manager 4.2. If necessary we will contact a statistician for data analyses (for example determining hazard ratios) Non-randomized studies will be analysed in exploratory. If a sufficient number of randomized trials is identified and included, the following subgroup analysis will be performed: clinical staging (stage A,B,C or stage I , II, III); pathological classification. Data from non-randomised studies for assessment of safety will be presented without summary statistics as an additional table, and discussed.

Subgroup analysis: If a sufficient number of randomized trials is identified and included, the following subgroup analysis will be performed: clinical staging (stage A,B,C or stage I , II, III); pathological classification.

Sensibility analysis: For dichotomous outcomes, patients with incomplete or missing data will be included in a sensitivity analysis by counting them as treatment failures to explore the possible effect of loss to follow-up on the findings ('worstcase' scenario). Heterogeneity will be tested for using Chi square with significance being set at $p<0.10$. Whenever there is significant heterogeneity, the random effects model will be used. Meta-analysis will be performed using the Cochrane software, RevMan Analysis, in Review Manager 4.2.

Country(ies) involved: China.

Keywords: Herbal medicine, meta-analysis, colorectal cancer, relapse and metastasis randomized controlled trials.

Contributions of each author:

Author 1 - Mo Tang.

Author 2 - Bin He.

Author 3 - Yufei yang.

Author 4 - Na Wu. 